\title{
Transmission of chlamydiae by the housefly
}

\author{
T. FORSEY AND S. DAROUGAR \\ From the Subdepartment of Virology, Department of Preventive Ophthalmology, Judd Street, \\ Institute of Ophthalmology, London WC1H $9 Q S$
}

SUMmARY The ability of the housefly to carry viable Chlamydia trachomatis and to transmit a chlamydial ocular infection was studied under laboratory conditions. After feeding flies (Musca domestica) on suspensions of egg yolk sac infected with $C$. trachomatis serotypes A or B (responsible for hyperendemic trachoma) the agents were reisolated from flies' intestines for up to 6 hours and from their legs and/or proboscises for up to 2 hours. It was found that the viability of chlamydiae is dependent on the protective effect of yolk concentration in the original inoculum. Results of experiments with guinea-pig inclusion conjunctivitis as an animal model show that under laboratory conditions flies can readily transmit this chlamydial ocular infection from one animal to another. These results suggest that under field conditions flies can play an important role in the transmission of trachoma, particularly in areas with favourable conditions such as a large reservoir of infection among children with severe trachoma, copious eye discharge caused by trachoma and associated bacterial infections, a large fly population, and close proximity of children in large family groups.

Flies have long been considered possible vectors of hyperendemic trachoma and associated bacterial conjunctivitis in the rural communities of developing countries. It has been noted that in these areas a seasonal increase in the prevalence of these infections coincides with an increase in the fly population. ${ }^{12}$ Recently Chlamydia trachomatis has been isolated in our laboratory from flies collected from around the eyes of children with active trachoma. ${ }^{3}$

Experimental attempts to define the role of the fly in transmission of the trachoma agent have yielded differing results. In 1962 Gear and colleagues ${ }^{4}$ fed flies of the species Musca sorbens on material infected with a trachoma agent but failed to reisolate the agent from these flies. Zardi in $1964^{5}$ claimed that, using flies of the species Musca domestica, he successfully reisolated a trachoma agent from flies between 4 to 15 days after feeding them with infected material.

In this investigation we have attempted to find out whether, under laboratory conditions, the housefly can carry viable trachoma agent and for how long, and using an animal model to determine whether eye-to-eye transmission of a chlamydial infection can be established by flies.

Correspondence to $\mathrm{Mr} \mathrm{T}$. Forsey.

\section{Materials and methods}

FLIES

The species Musca domestica (common housefly) was used in this study. Puparia were externally disinfected by washing with $5 \%$ phenol and placed in sterile gauze cages. They were maintained under clean conditions in a laminar flow cabinet. On emergence adult flies were fed on solid sucrose and a $50 \%$ skimmed milk/water mixture to which formaldehyde at 1:10000 had been added as a preservative. Each cage contained approximately 200 adult flies at the start of each experiment.

\section{CHLAMYDIAL AGENTS}

Chlamydia trachomatis serotypes A and B responsible for hyperendemic trachoma ${ }^{6} 7$ were used to contaminate flies. The agents were inoculated into the yolk sacs of fertile hens' eggs, and on reaching a $50 \%$ death rate the live eggs were harvested. Yolk sacs from these eggs were homogenised with sucrose phosphate buffer $(2 \mathrm{SP})^{8}$ to produce $40 \%$ or $10 \%$ suspensions. On titration in irradiated McCoy cells the $40 \%$ suspensions were found to contain approximately $10^{6}$ inclusion forming units per millilitre (IFU/ml).

For the animal model experiments the guinea-pig inclusion conjunctivitis (GPIC) agent, ${ }^{9}$ a Chlamydia 
psittaci strain, was grown in the same way and infected yolk sacs processed as $50 \%$ suspensions. These contained approximately $10^{7} \mathrm{IFU} / \mathrm{ml}$.

All yolk sac suspensions were stored at $-70^{\circ} \mathrm{C}$ until used.

\section{CONTAMINATION OF FLIES}

Food was removed from a cage and the flies were starved overnight. Infected inoculum consisting of $40 \mathrm{ml}$ of yolk sac suspension poured on to sterile lint in a Petri dish was introduced into the cage. The ambient temperature was $22-25^{\circ} \mathrm{C}$. Flies were allowed to feed for the required time, and the inoculum was replaced by normal food. Flies were collected at various time intervals in batches of approximately 15 flies by means of a sterile glass tube attached to a vacuum pump. The collection tube was washed and disinfected with $70 \%$ alcohol between batches to avoid cross-contamination. Flies were killed by cooling to $-70^{\circ} \mathrm{C}$.

Each fly was dissected to collect the intestines and legs plus proboscis separately. Intestines and legs/ proboscises from each batch were placed in separate plastic vials containing 2SP. Positive control specimens were collected to test the viability of the $C$. trachomatis suspension by gently pressing cottonwool swabs on to the infected lint and placing them directly into vials of 2 SP.

All specimens were stored at $-70^{\circ} \mathrm{C}$ prior to inoculation in cell culture.

\section{ISOLATION OF Chlamydia trachomatis}

Chlamydial isolation was carried out by inoculating monolayers of irradiated McCoy cells following the method of Darougar et al $^{10}$. The contents of each vial (intestines or legs/proboscises) were first macerated with sterile Pasteur pipettes and shaken with glass beads for 30 seconds. The supernatant was withdrawn, mixed with $2 \mathrm{ml}$ of cell culture medium, ${ }^{8}$ centrifuged at $1500 \mathrm{~g}$ for 10 minutes, and the supernatant used to inoculate 2 tubes of McCoy cells in the usual way. After approximately 60 hours' incubation the cell monolayers were fixed, stained with Giemsa, and screened for chlamydial inclusions under dark-ground illumination.

\section{ANIMAL MODEL EXPERIMENTS}

Six healthy female albino guinea-pigs (Dunken Hartley strain) were used. Two guinea-pigs were inoculated with 1 drop (approximately $0.05 \mathrm{ml}$ ) of GPIC agent suspension in the eye. After 4 days a severe conjunctivitis, with mucoid discharge, developed in both eyes of each animal. Infection was confirmed by detecting GPIC agent inclusions in Giemsa stained conjunctival scrapings. The heads of the infected guinea-pigs were then introduced into cages containing uninfected flies and exposed for 1 hour. These flies were then transferred to fresh, sterile cages, and the eyes of uninfected guinea-pigs were exposed to these flies. Two animals were exposed to each cage of flies consecutively for 1 hour each.

Exposure of guinea-pigs to flies was facilitated by immobilising the animal's body in a box with the head protruding through a hole cut in one end. Fresh boxes were used for each animal, and the guinea-pigs were subsequently maintained in separate cages to avoid cross-contamination. The eyes of the animals were examined daily for up to 3 weeks after exposure under an operating microscope. The clinical findings were scored and recorded following the method described previously. ${ }^{11}$ Conjunctival scrapings were also collected for the detection of GPIC agent inclusions.

\section{Results}

In an initial experiment flies were fed for a period of 2 hours on a $40 \%$ infected yolk sac suspension of C. trachomatis TRIC serotype B. Chlamydiae were isolated from the legs/proboscises and intestines of these flies during the period of feeding and for up to 4 hours after removal of the inoculum (Table 1). No attempts were made to count the number of inclusions obtained from flies in this experiment.

Subsequently flies were fed for a period of 22 hours on a $40 \%$ yolk sac suspension of $C$. trachomatis TRIC serotype A. Chlamydiae were isolated from the contaminated lint for up to 32 hours (Table 2). The numbers of inclusions obtained in these specimens are shown in Table 2. From flies fed on this inoculum chlamydiae were isolated from the legs/proboscises during feeding but not after removal of the inoculum, whereas from the intestines chlamydiae were isolated for up to 28 hours ( 6 hours after removal of the inoculum) (Table 2). The

Table 1 Isolation of chlamydiae from flies fed for 2 hours on a $40 \%$ suspension of yolk-sac infected with C. trachmomatis TRIC serotype B

\begin{tabular}{|c|c|c|c|c|c|c|c|}
\hline \multirow[t]{2}{*}{ Specimen } & \multicolumn{7}{|c|}{ Time (hours) } \\
\hline & 0 & 2 & 3 & 4 & 5 & 6 & 7 \\
\hline Legs/proboscises & $-\mathbf{a}$ & $+b$ & + & + & - & - & - \\
\hline Intestines & - & + & + & + & + & + & - \\
\hline $\begin{array}{l}\text { Positive control } \\
\text { swabs }\end{array}$ & & + & + & + & + & + & + \\
\hline & $\begin{array}{l}\text { Inoculum } \\
\text { introduced }\end{array}$ & $\begin{array}{l}\text { Inoc } \\
\text { rem } \\
2 \mathrm{hr}\end{array}$ & & & & & \\
\hline
\end{tabular}


Table 2 Isolation of chlamydiae from flies fed for 22 hours on a $40 \%$ suspension of yolk sac infected with C. trachomatis TRIC serotype A

\begin{tabular}{|c|c|c|c|c|c|c|c|c|c|}
\hline \multirow[b]{2}{*}{ Specimen } & \multicolumn{9}{|c|}{ Time (hours) } \\
\hline & 0 & 4 & 8 & 16 & 24 & 28 & 32 & 36 & $\begin{array}{l}\text { Daily up to } \\
\text { day } 10\end{array}$ \\
\hline Legs/proboscises & - & $+(1)^{\mathrm{a}}$ & $+(2)$ & $+(>1000)$ & - & - & - & - & 一 \\
\hline Intestines & 一 & - & $+(6)$ & $+(>1000)$ & $+(1)$ & $+(1)$ & - & - & - \\
\hline Positive control swabs & & $+(>1000)$ & $+(>1000)$ & $+(50)$ & $+(10)$ & $+(6)$ & $+(3)$ & - & - \\
\hline & Ino & & & & $\begin{array}{l}\text { noculum } \\
\text { emoved } \\
2 \mathrm{hr}\end{array}$ & & & & \\
\hline
\end{tabular}

number of inclusions obtained from flies during feeding was over 1000 per coverslip (at 16 hours), whereas after removal of the inoculum only 1 inclusion was obtained from each intestinal specimens (Table 2).

By means of a $10 \%$ yolk sac suspension of $C$. trachomatis TRIC serotype A, chlamydiae were isolated from the contaminated lint and from the legs/proboscises of flies fed on this inoculum, for up to 8 hours of feeding period (Table 3 ). In this experiment no isolate was obtained from the intestines. The numbers of inclusions obtained from the lint and infected flies are shown in Table 3.

In 2 separate experiments a total of 4 healthy guinea-pigs ( 2 for each experiment) were exposed to flies which had been in contact with the eyes of guinea-pigs infected with GPIC agent. Each animal was exposed to the flies for a period of 1 hour. In both experiments the 2 guinea-pigs which were exposed to the flies for the first hour developed a moderate conjunctivitis 8 and 10 days after exposure. Of the 2 guinea-pigs which were exposed subsequently (second hour) one developed a mild conjunctivitis after an incubation period of 18 days and the other one remained uninfected. Conjunctival scrapings from these animals with conjunctivitis contained typical GPIC agent inclusions.

\section{Discussion}

Isolation of $C$. trachomatis from the lint contamininated with $10 \%$ or $40 \%$ yolk sac suspension of the agent shows that at an ambient temperature of $22-25^{\circ} \mathrm{C} \mathrm{C}$. trachomatis can sustain its infectivity between 8 and 32 hours, the time being dependent on the concentration of the yolk sac suspension (Tables 2 and 3). No marked difference in the number of inclusions was found in specimens collected from these 2 suspensions at 8 hours (Table 2), but at 16 hours only those specimens which were collected from a $40 \%$ suspension were positive. This finding suggests that a higher concentration of yolk material in the $40 \%$ suspension protects chlamydiae more efficiently. This result was confirmed by a separate experiment in our laboratory when we found that the addition of yolk material to transport medium ( 2 to $5 \% \mathrm{v} / \mathrm{v}$ ) improved the protective effect of the medium during transport and storage of chlamydial agents (unpublished observations).

The results of the present study show that flies are capable of carrying infectious chlamydial particles on their legs/proboscises and in their intestines for a short period of 2 to 6 hours (Table 1 and 2). The efficacy of flies in transmitting infectious chlamydiae

Table 3 Isolation of chlamydiae from flies fed for 22 hours on a $10 \%$ suspension of yolk sac infected with C. trachomatis TRIC serotype A

\begin{tabular}{|c|c|c|c|c|c|c|c|c|c|}
\hline \multirow[b]{2}{*}{ Specimen } & \multicolumn{9}{|c|}{ Time (hours) } \\
\hline & 0 & 4 & 8 & 16 & 24 & 28 & 32 & .36 & $\begin{array}{l}\text { Daily up to } \\
\text { day } 10\end{array}$ \\
\hline Legs/proboscises & - & $+(1)$ & $+(2)$ & - & - & - & - & - & 一 \\
\hline Intestines & - & - & - & - & - & - & 一 & - & 一 \\
\hline Positive control swabs & & $+(>1000)$ & $+(>1000)$ & - & 一 & - & - & - & - \\
\hline
\end{tabular}


appears to depend on factors such as concentration and infectivity of chlamydial agents in contaminated material, feeding habits of flies, and exposure of chlamydiae to environmental factors during transmission. We found that flies transmit chlamydiae for a longer period when the particles are protected by a higher concentration of yolk material. In the case of trachoma the presence of mucoid or mucopurulent discharge in the eye may have a similar role in protecting chlamydiae shed from the eye. Studies by Darougar et al. ${ }^{12}$ showed that the chlamydial isolation rate from eye secretions of patients with trachoma is directly related to the severity of conjunctival inflammatory responses, to the number of chlamydial inclusions present in their conjunctivae, and to the amount of mucoid discharge in their eyes. They suggested that patients with moderate to severe trachoma are a reservoir of infectious agents and that excessive amounts of discharge in the eye (produced by trachoma or associated bacterial conjunctivitis) protect the chlamydiae and thus facilitate transmission of the agent by flies.

In the rural communities of developing countries eye-seeking flies, especially Musca sorbens, are attracted to eye discharges and avidly feed on them. During the course of this study and our work in the field we observed that flies alight at the eyes or on inocula repeatedly but feed for only short periods of 30 to 60 seconds on each occasion. The frequency of flies landing at the eyes coupled with their particular mechanism of feeding, which involves vomiting, enables flies to transfer ocular discharge from one eye to another rapidly ${ }^{3}$ and to accumulate and build up higher doses of infectious chlamydiae (Table 2).

Chlamydiae were isolated from legs/proboscises of flies for a period of 2 hours and from the intestine for a period of 6 hours after feeding on infected material. This difference may be due to the fact that on legs/proboscises contaminated material is directly exposed to environmental factors such as dryness and heat, which are known to rapidly inactivate chlamydiae.

Failure to isolate chlamydiae from the intestines of flies after 6 hours suggests that chlamydiae do not replicate in the intestinal tissues of flies. This finding does not support the results of Zardi, ${ }^{5}$ who fed flies for 3 days on infected inoculum and claimed that, using an egg culture system, he isolated chlamydiae from these flies on days 4 to 15 after feeding.

Results of the experiments with an animal model show that under favourable laboratory conditions flies can readily transmit an ocular chlamydial infection from one animal to another. In the field we have isolated chlamydiae from only a few flies which were feeding at the eyes of children with moderate to severe trachoma. ${ }^{3}$ This result suggests that in the field flies can pick up chlamydiae while feeding on eye discharge, but the adverse effects of environmental factors, particularly heat and dryness, probably inactivate the agent. However, this fact is countered by the large reservoir of infection in young children with moderate to severe trachoma, the presence of copious mucoid and mucopurulent discharges (caused by trachoma and associated bacterial infections), the high population of flies, particularly Musca sorbens, feeding freely at the eyes of babies and children, and the close proximity of children in large family units in the rural communities. Further studies are required to determine the precise importance of flies in the transmission of trachoma.

We thank Dr J. F. Invest, Wellcome Research Laboratories, for supplying Musca domestica pupae, Professor Barrie R. Jones for his advice, and an anonymous donor for financial support.

\section{References}

1 Wilson RP. Annual Report of the Memorial Ophthalmic Laboratory, Giza, Cairo. Giza Ophthalm Lab Rep 1936; 11: $95-112$.

2 Greenberg B. Flies and Diseases. New Jersey: Princeton University Press, 1973; 2.

3 Jones BR. The prevention of blindness from trachoma. Trans Ophthalmol Soc UK 1975; 95: 16-33.

4 Gear J, Cuthbertson E, Ryan J. A study of South African strains of trachoma in experimental animals. Ann $N Y$ Acad Sci 1962; 98: 197-200.

5 Zardi $O$. The importance of Musca domestica as an agent in the transmission of trachoma. Nouvi Annali d'Igiene e Microbiologica 1964; 6: 587-90.

6 Wang S-P, Grayston JY. Immunologic relationship between genital TRIC, lymphogranuloma venereum and related organisms in a new microtitre indirect immunofluorescence test. Am J Ophthalmol 1970; 70: 367-74.

7 Treharne JD, Darougar S, Jones BR. Modification of the micro-immunofluorescence test to provide a routine serodiagnostic test for chlamydial infection. $J$ Clin Pathol 1977; 30: 510-7.

8 Gordon FB, Harper IA, Quan AL, Treharne JD, Dwyer R St C, Garland JA. Detection of Chlamydia (Bedsonia) in certain infections in man. 1. Laboratory procedures: Comparison of yolk-sac and cell culture for detection and isolation. J Infect Dis 1969; 120: 451-62.

9 Murray ES. Guinea pig inclusion conjunctivitis virus. 1. Isolation and identification as a member of the psittacosislymphogranuloma group. J Infect Dis 1964; 114: 1-12.

10 Darougar S, Kinnison JR, Jones BR. Simplified irradiated McCoy cell culture for isolation of Chlamydiae. In: Nichols RL, ed. Trachoma and Related Disorders. Amsterdam: Excerpta Medica 1970: 63-70.

11 Monnickendam MA, Darougar S, Treharne JD, Tilbury AM. Guinea-pig inclusion conjunctivitis as a model for the study of trachoma: clinical, microbiological, serological, and cytological studies of primary infection. $\mathrm{Br}$ J Ophthalmol 1980; 64: 279-83.

12 Darougar S, Forsey T, Jones BR, Allami J, Houshmand A. Isolation of Chlamydiae trachomatis from eye secretions (tears). Br J Ophthalmol 1979; 63: 256-8. 